PO019

\title{
REPRESENTATIVE COLOR OF THE WHOLE-FACE IMAGE AND ITS RELATION TO FINISH-UP IMPRESSION \\ Kohei Kawame et al.
}

DOI 10.25039/x46.2019.PO019

from

CIE x046:2019

Proceedings

of the

29th CIE SESSION

Washington D.C., USA, June 14 - 22, 2019

(DOI 10.25039/x46.2019)

The paper has been presented at the 29th CIE Session, Washington D.C., USA, June 14-22, 2019. It has not been peer-reviewed by CIE.

(C) CIE 2019

All rights reserved. Unless otherwise specified, no part of this publication may be reproduced or utilized in any form or by any means, electronic or mechanical, including photocopying and microfilm, without permission in writing from CIE Central Bureau at the address below. Any mention of organizations or products does not imply endorsement by the CIE.

This paper is made available open access for individual use. However, in all other cases all rights are reserved unless explicit permission is sought from and given by the $\mathrm{CIE}$.

CIE Central Bureau

Babenbergerstrasse 9

A-1010 Vienna

Austria

Tel.: +4317143187

e-mail: ciecb@cie.co.at

www.cie.co.at 


\title{
REPRESENTATIVE COLOR OF THE WHOLE-FACE IMAGE AND ITS RELATION TO FINISH-UP IMPRESSION
}

\author{
Kawame, K. ${ }^{1}$, Shiromizu, S. ${ }^{1}$, Arimoto, K. ${ }^{3}$, Kimura, M. ${ }^{3}$, Hata, H. ${ }^{3}$, Koshino, M. ${ }^{3}$, \\ Ishikawa, T. ${ }^{1}$ Ayama, M. ${ }^{1,2}$, \\ ${ }^{1}$ School of Engineering, Utsunomiya University, JAPAN \\ ${ }^{2}$ Center for Optical Research \& Education, Utsunomiya University, JAPAN \\ ${ }^{3}$ Shiseido Global Innovation Center, JAPAN \\ mt186708@cc.utsunomiya-u.ac.jp
}

DOI 10.25039/x46.2019.PO019

\begin{abstract}
In recent studies on the evaluation of skin color of facial image, colorimetric values of whole face are used to indicate facial color, since 2D-colorimeter being developed and commercially available. However, what kind of colorimetric values derived from all pixels in skin color area are appropriate has not been examined precisely. The purpose of the study is to determine an appropriate representative colorimetric values of skin color in the whole-face image. Average $\left(\mathrm{L}^{*}, \mathrm{a}^{*}, \mathrm{~b}^{*}\right)$ values of the top $60 \%$ in the appearance-frequency in the skin color area is proposed as a good representative color of the whole-face image. The area of acceptable "Finish-up impression" in the CIELAB space is indicated using these colorimetric values.
\end{abstract}

Keywords: Skin color, Facial image, Colorimetric values, 2D-colorimeter, Finish-up impression,

\section{Introduction}

In recent studies on the evaluation of skin color of facial image, colorimetric values of whole face are often used to indicate facial color, since 2D-colorimeter being developed and commercially available. In some of previous studies [1,2], average values are used probably due to statistical expediency. In some cases, the way to derive colorimetric values was not fully described [3-5]. What kind of colorimetric values are appropriate that are derived from all pixels in skin color area, has not been examined precisely. In most of the studies on facial color, CIELAB values are used to represent the stimulus color [1,2,6-9]. There are various ways to obtain $\left(L^{*}, a^{*}, b^{*}\right)$ value, for example, of each pixel, when using an image on a display. In an extreme case, they can be calculated from RGB pixel data without any colorimetry of the display. From visual science point of view, colorimetric values of the light inserting to the eye under the observing condition is the most important, and should be employed. At the present, the values measured using a 2D-colorimeter is most direct and reliable. But then the next problem is what kind of colorimetric values derived from or based on all pixels in skin color area are the most appropriate to represent the skin color of facial image. This issue is still being discussed in various fields relating to color science and lighting engineering using $2 \mathrm{D}$ colorimeter. Thus the purpose of the study is to determine an appropriate representative colorimetric values of skin color in the whole-face image.

\section{Experiment}

\subsection{Test Stimuli}

A facial image of 3 Japanese women in her twenties without makeup was taken by a digital camera and set as an original image. We call them test faces $A, B$, and $C$, in the present study. For each of the test faces, image group composed of 343 was created in which the $L^{*}, a^{*}$, and $b^{*}$ of each pixel in the skin color area were changed with nearly a constant step of $\Delta L^{*}=2, \Delta a^{*}=2$, and $\Delta b^{\star}=2$, from -6 to 6 along 3 axes.

\subsection{Colorimetry}

For all the test stimuli, skin color part was extracted by deleting the eyes, eyebrows, and lips as shown in Figure 1, where the original and several extreme stimuli are indicated. 
Chromaticities and luminance of these images on the display were measured using 2D colorimeter (Konica Minolta CA-2500) with the resolution of $980 \times 980 . L^{*} a^{*} b^{*}$ values of each pixel of, not the original test image, but the image taken by the 2D colorimeter, are obtained employing the maximum white of the display as the reference white, for each of 119 test images nearly evenly selected from 343 test images.

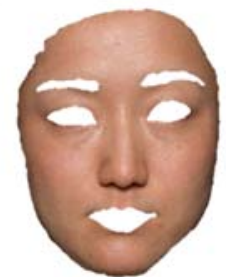

$(0,0,0)$

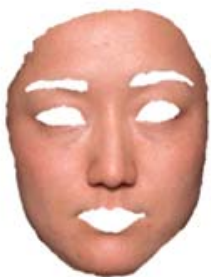

$(6,-6,0)$

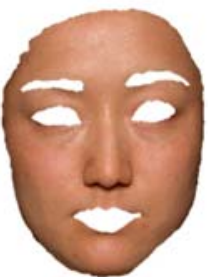

$(-2,4,6)$

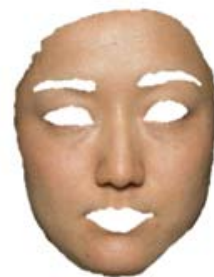

$(4,-6,2)$

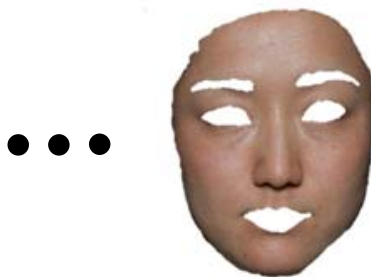

$(-6,-6,-6)$

Figure 1 - Examples of the images of facial skin area used for the 2D colorimetry. Test face $A$. Numbers below are nominal values of $\left(\Delta L^{*}, \Delta a^{*}, \Delta b^{*}\right)$.

\subsection{Subjective Evaluation}

Subjective evaluation experiments are carried out for the words of "Finish-up impression", "Blendable to her skin" "Difference between face and neck color", "Brightness or darkness", and "Redness or yellowness" [10]. Details of rating procedure are described in that study.

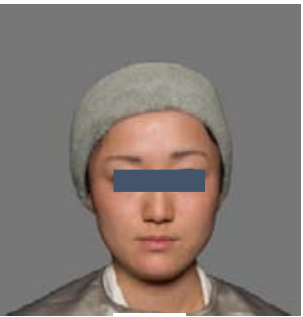

A

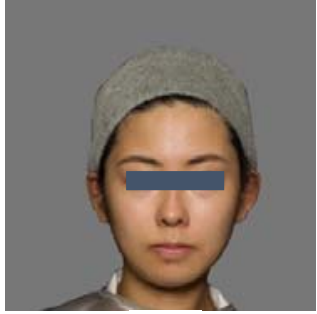

B

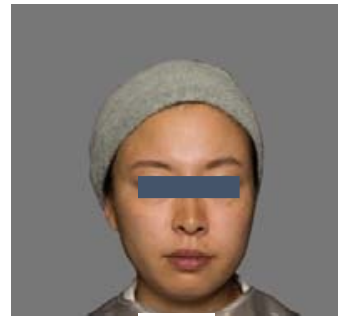

C

Figure 2 - Example of test image in subjective evaluations. Bars were not presented in the experiment.

\section{Appearance-frequency}

\subsection{Appearance-frequency and distribution map}

At first, we wanted to grasp the distribution of pixels of each test stimulus in the CIELAB color space. So, a histogram of appearance-frequency of $\left(L^{*}, a^{*}, b^{*}\right)$ is created for each of 119 test images. The principle of calculation and the resulted histogram of the original image of the test face $A\left(\Delta \mathrm{L}^{*}, \Delta \mathrm{a}^{*}, \Delta \mathrm{b}^{*}\right)=(0,0,0)$ is shown in Figure 3 . We divided the CIELAB space into the small cubes of which length of one side is 1 . We call it 'voxel', and count the number of pixels in each voxel for all pixels in the skin color area of the image. This means that the distribution of pixels in the CIELAB space is obtained. Horizontal and vertical axes of the histogram indicate $\left(L^{*}, a^{*}, b^{*}\right)$ of the point of the voxel closest to the origin, and the number of pixels, respectively. For the original image of test face $A$, the most dense voxel is the one shown in the right-hand side of Figure 3 . Then the average values of top $x \%$ are calculated that are denoted as $\left(L^{*}\right.$ avx, $\left.a^{*} a v x, b^{*} a v x\right)$.

Secondly, we wanted to know the locations of the pixels of high appearance-frequency in the face. So, all pixels of which $\left(L^{*}, a^{*}, b^{*}\right)$ is included in $\mathrm{x} \%$ appearance-frequency group were plotted on the face. We call the figure a 'distribution map', created the maps of the top $10 \%$ to $100 \%$ with a step of $10 \%$. Figure 4 shows the distribution maps of $30 \%, 60 \%$, and $100 \%$. Thus $\left(L^{*}{ }_{a v x}, a^{*} a v x, b_{a v x}\right)$ of $100 \%$ is equal to the average of skin color area. As shown in Figure 4 , pixel distribution of the top $30 \%$ is relatively localized, while that of the top $60 \%$ seems to nearly 
uniformly distribute over the whole face, excluding glossy part in the forehead, nose, or cheeks, and the shaded areas along the outside boundary of the face.

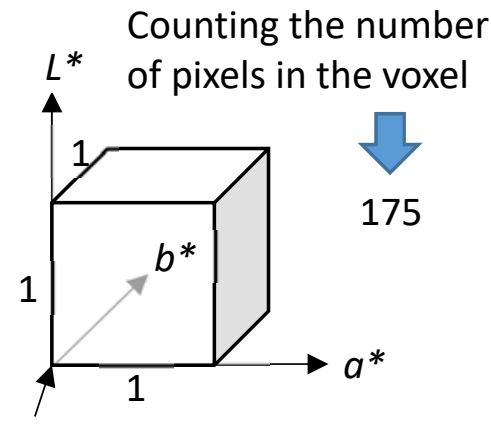

$(63,28,27)$

Test face $A$, original image

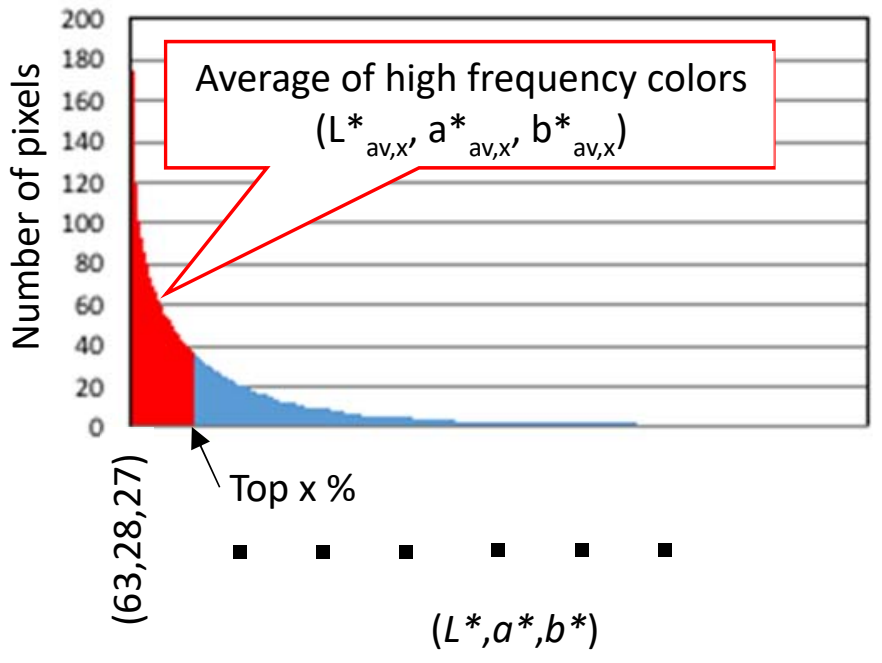

Figure 3 - The principle of calculation (left) and the resulted histogram (right).

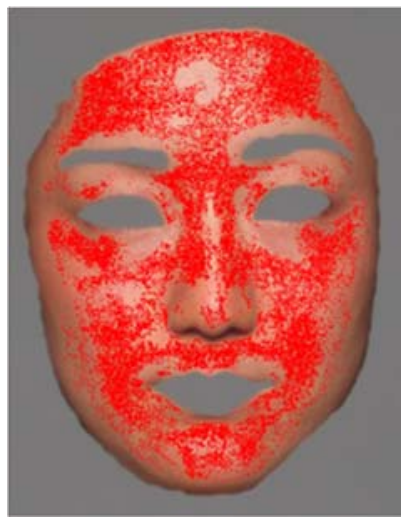

$30 \%$

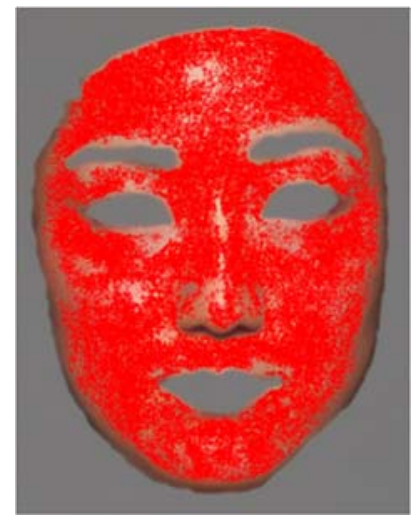

$60 \%$

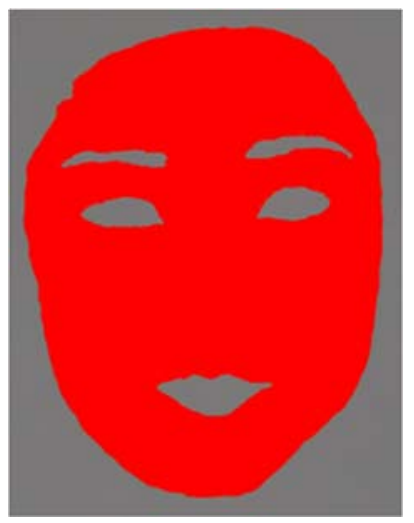

$100 \%$

Figure 4 - Distribution map of the top $30 \%, 60 \%$, and $100 \%$. Test face $A$.

As the first approach to propose an appropriate representative colorimetric values of skin color of facial image, the change $L^{*} a v, a^{*} a v$, and $b^{\star}{ }_{a v}$ against the percentage was analysed. Results of $L^{*}{ }_{a v}$ for 119 test stimuli are indicated in Figure 5. Black circles denote the grand average of them. As the percentage increases, $L^{*}$ av decreases because the number of low $L^{*}$ pixels, especially in the outside boundary, increases. The slope of $L^{*}$ av between adjacent $\%$ is plotted in Figure 6 . They are all negative except between $10 \%$ and $20 \%$. Value of slope stays around 1 between $30-40 \%$ to $60-70 \%$, indicating that the decrease of $L{ }^{*}$ av is shallow within these range. Absolute value of the slope begins to increase from $70-80 \%$ and becomes up to -4 at $90-100 \%$ indicating that the sharp decrease of $L^{*}$ av. The change of the slope of $a^{*}{ }_{a v}$ and $b^{*} a v$ values indicated quite similar tendency. The same analysis was also done for the test faces $B$ and $C$. No significant differences were observed among different test faces. Therefore, we propose the average values of luminance and chromaticity of $\left(L^{*}, a^{*}, b^{*}\right)$ values of the top $60 \%$ in the appearance-frequency as a representative color of the whole-face image. It is denoted as $\left(L^{*}{ }_{a v, 60, i}, a^{*}{ }_{a v, 60, i}, b^{*}{ }_{a v, 60, i}\right)$, where $i$ indicates the test image number. 


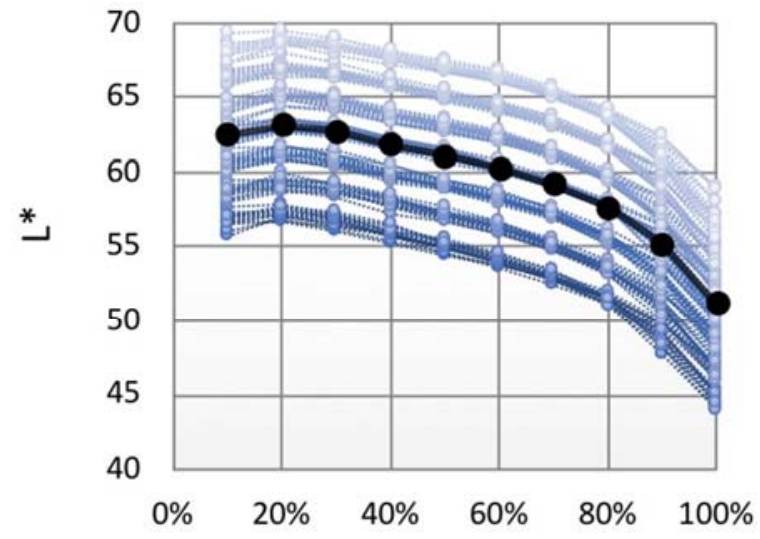

The top $\%$ of the appearance-frequency

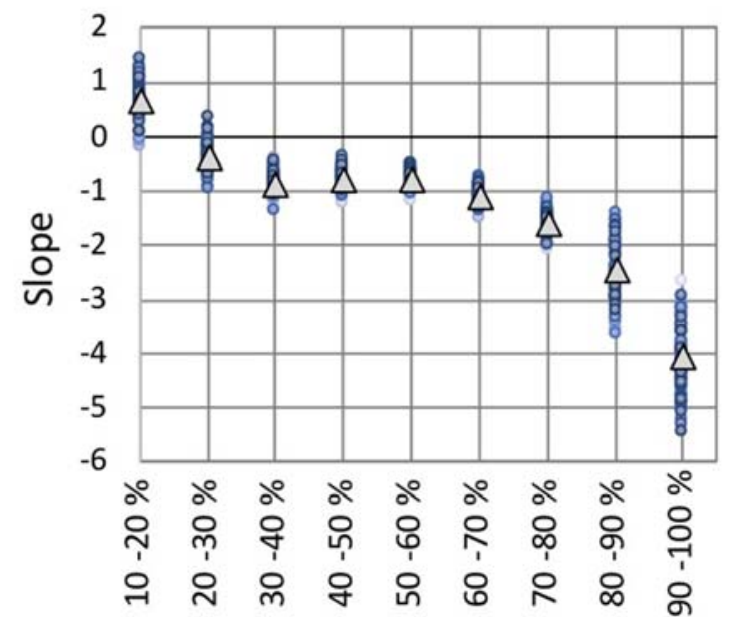

Figure 6 - Change of the slope in the curves in Figure 5. Triangles denote the slope of the grand average in Figure 5.
Figure 5 - Change of $L^{*}$ av against the top percentage of the appearance-frequency for 119 test images. Black circles denote grand average. Test face $A$.

\section{Relation to "Finish-up impression" evaluation}

Rating scores of "Finish-up impression" evaluation were plotted in the CIELAB space using $\left(L^{*}{ }^{*}, 60, i, a^{*}{ }_{a v}, 60, i, b^{*}{ }_{a v, 60, i}\right), i=1$ to 119 , and the contour lines were drawn in the planes of the same nominal value of $\Delta L^{*}$. Results at $\Delta L^{*}=4$ for the test faces $\mathrm{A}, \mathrm{B}$, and $\mathrm{C}$ are shown in Figure 7. Note that the range of axes are different among test faces. olorimetric values of the images that obtained high scores commonly among observers were plotted in the $L^{*} v s C^{*}$ graph. All results locate in the larger $L^{*}$ area than the original image for both test faces $A$ and $B$, while the areas along the $C^{*}$ axis slightly differ between the test faces $A$ and $B$. In the case of the test face $A$, all results locate smaller $C^{*}$ area than the original image, but for the test face $B$, that tendency is not clearly observed.

\subsection{Relation to subjective evaluation of "Finish-up impression"}

Average rating score of subjective evaluation of "Finish-up impression" was plotted in the CIELAB space at the point of $\left(L^{*}{ }_{a v, 60, i} a^{*}{ }^{*}, 60, i, b^{*}{ }^{*}, 60, i\right), i=1$ to 119 , and the contour maps of equal score in the $a^{*}-b^{*}$ plane were drawn at each of the nominal $\Delta L^{*}$ levels from $\Delta L^{*}=-6$ to 6 . Contour maps in the $\Delta L^{*}=4$ for the test faces $A, B$, and $C$ are indicated in Figure 7 . For all test faces, the highest scores were obtained in the $\Delta L^{*}=4$. Note that the range of axes are different among test faces. High score area is close to the $\left(a^{*}, b^{*}\right)$ position of the original image. Shape of contours is ellipsoidal with the long axis along radial direction. 

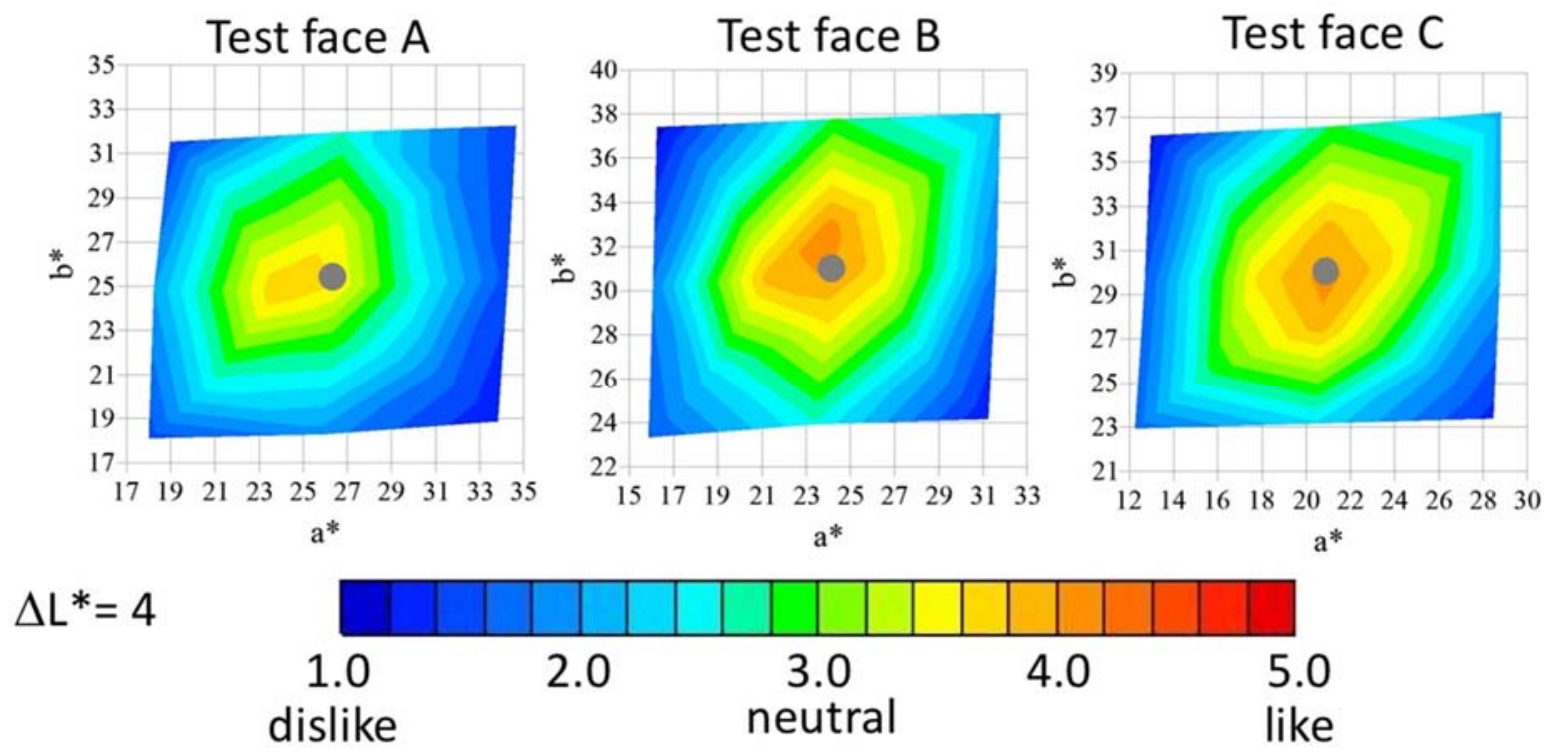

Figure 7 - Contour maps of the rating scores for "Finish-up impression" in the $a^{*}-b^{*}$ plane for images of the test faces $A, B$, and $C$, drawn based on $\left(L_{a v, 60}^{*}, a_{a v, 60}^{*}, b_{a v, 60}^{*}\right)$. Nominal value of $\Delta L^{*}$ is 4 . Gray circles denote the $\left(a_{a v, 60}^{*}, b_{a v, 60}^{*}\right)$ of the original test image.

\section{Conclusion}

To determine an appropriate representative color of the whole-face image, $L^{*} a^{*} b^{*}$ values of the whole-face images were calculated based on the $2 \mathrm{D}$ colorimeter measurement. A histogram of appearance-frequency of $\left(L^{*}, a^{*}, b^{*}\right)$ was created, and the average values were calculated from the top $10 \%$ to $100 \%$ with a step of $10 \%$. Based on the slope of decrease of $L^{*}, a^{*}$, and $b^{*}$, average values of the top $60 \%$ in the appearance-frequency seem a good representative color of the whole-face image, that is neither strongly affected by extremely dark pixels in the outside border, nor extremely bright pixels in the cheek, nose, and/or forehead.

\section{References}

1. Yoshikawa, H. et al. 2012. Effect of Chromatic Components on Facial Skin Whiteness. Color Res. Appl., 37, 281-291.

2. Zeng, H. Luo, R. 2013. Colour and Tolerance of Preferred Skin Colours on Digital Photographic Images. Color Res. Appl., 38, 30-45.

3. Fink, B. et al. 2001. Human (Homo sapiens) Facial Attractiveness in Relation to Skin Texture and Color. J. Comp. Psychol., 115, 92-99.

4. Fink, B., Matts, P. J. 2008. The Effects of Skin Colour Distribution and Topography Cues on the Perception of Female Facial Age and Health. J. Eur. Acad. Darmatol. Venereol., 22, 493-498.

5. Samson, N. et al. 2011. Interaction of Skin Color Distribution and Skin Surface Topography Cues in the Perception of Female Facial Age and Health. J. Cosmet. Dermato., 10, 78-84.

6. Zhao, B. et al. 2017.Developing a Model for Predicting Whiteness of Human Face. Proceedings of the $13^{\text {th }}$ AIC Congress 2017, USB.

7. Zhao, B. et al. 2017. Investigation of Facial Attractiveness Using a Facial Image Database. Proceedings of the $13^{\text {th }}$ AIC Congress 2017, USB.

8. Wang, M. et al., 2017.The Impact of Skin Colour on Facial Impressions. Proceedings of the $13^{\text {th }}$ AIC Congress 2017, USB.

9. Shiromizu, S. et al. 2018. KANSEI Evaluation of Skin Color of Young Japanese Women on a Display and the Relation to Colorimetric Values of Whole Face. Proceedings of IDW2018, 960-963.

10. Ayama, M. et al. 2019. Colorimetric Values of Image Skin Color in the Whole Face and Cheek Part, and Their Relation to Subjective Evaluation. Proceedings of CIE2019. 